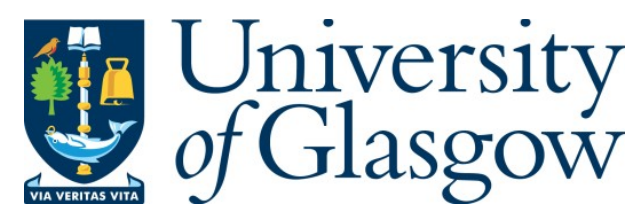

Afzal, A., Zaidi, S. A. R., Shakir, M. Z., Imran, M. A., Ghogho, M., Vasilakos, A. V., McLernon, D. C., and Qaraqe, K. (2015) The cognitive internet of things: a unified perspective. Mobile Networks and Applications, 20(1), pp. 72-85.

There may be differences between this version and the published version. You are advised to consult the publisher's version if you wish to cite from it.

http://eprints.gla.ac.uk/132812/

Deposited on: 14 December 2016

Enlighten - Research publications by members of the University of Glasgow http://eprints.gla.ac.uk 


\title{
The Cognitive Internet of Things: A Unified Perspective
}

\author{
Asma Afzal - Syed Ali Raza Zaidi - Muhammad Zeeshan Shakir • \\ Muhammad Ali Imran - Mounir Ghogho - Athanasios V. Vasilakos • \\ Des McLernon · Khalid Qaraqe
}

Received: - / Accepted: -

\begin{abstract}
In this article, we present a unified perspective on the cognitive internet of things (CIoT). It is noted that within the CIoT design we observe the convergence of energy harvesting, cognitive spectrum access and mobile cloud computing technologies. We unify these distinct technologies into a CIoT architecture which provides a flexible, dynamic, scalable and robust network design road-map for large scale IoT deployment. Since the prime objective of the CIoT network is to ensure connectivity between things, we identify key metrics which characterize the network design space. We revisit the definition of cognition in the context of IoT networks and argue that both the energy efficiency and the spectrum efficiency are key design constraints. To this end, we define a new performance metric called the 'overall link success probability' which encapsulates these constraints. The overall link success probability is characterized by both the self-sustainablitiy of the link through energy harvesting and the availability of spectrum for transmissions. With the help of a reference scenario, we demonstrate that well-known tools
\end{abstract}

A. Afzal · S. A. R. Zaidi · D. C. McLernon · M. Ghogho Department of Electronic and Electrical Engineering, University of Leeds, Leeds LS2 9JT, United Kingdom. E-mail: \{elaaf, elsarz, d.c.mclernon, m.ghogho@leeds.ac.uk\}

M. Shakir

K. Qaraqe

Texas A\&M University, Doha, Qatar. E-mail: \{muhammad.shakir@qatar.tamu.edu\}

M. A. Imran

University of Surrey, Guildford, United Kingdom. E-mail: \{m.imran@surrey.ac.uk\}

A. V. Vasilakos

Kuwait University, Kuwait. E-mail: \{vasaliko@ath.forthnet.gr\} from stochastic geometry can be employed to investigate both the node and the network level performance. In particular, the reference scenario considers a large scale deployment of a CIoT network empowered by solar energy harvesting deployed along with the centralized CIoT device coordinators. It is assumed that CIoT network is underlaid with a cellular network, i.e., CIoT nodes share spectrum with mobile users subject to a certain co-existence constraint. Considering the dynamics of both energy harvesting and spectrum sharing, the overall link success probability is then quantified. It is shown that both the self-sustainability of the link, and the availability of transmission opportunites, are coupled through a common parameter, i.e., the node level transmit power. Furthermore, provided the co-existence constraint is satisfied the link level success in the presence of both the inter-network and intra-network interference is an increasing function of the transmit power. We demonstrate that the overall link level success probability can be maximized by employing a certain optimal transmit power. Characterization of such an optimal operational point is presented. Finally, we highlight some of the future directions which can benefit from the analytical framework developed in this paper.

Keywords Internet-of-Things - Cognitive Radios · Solar Energy Harvesting - Stochastic Cloud Cover . Shared Spectrum · Underlay · Interference

\section{Introduction}

The term 'internet-of-things' (IoT) was coined by Kevin Ashton in 1999. The central idea was to empower everyday objects with internet connectivity thus enabling pervasive and autonomous communication. The foundation of IoT is based on Weiser's [1] vision of profound 
software/hardware technologies that weave themselves into the fabric of everyday life such that they become indistinguishable. The functionality and modalities of these technologies is distributed across a variety of interconnected objects. This inter-connectivity of these objects is pivotal as the collective intelligence of the IoT network emerges from simple object level interactions. In turn, such a collective intelligence can be credited with driving significant innovations in the context of various applications under the umbrella of smart homes and cities.

\subsection{The IoT Grand Challenge}

A recent survey from EiU [2] indicated that around $75 \%$ of businesses are either actively considering or employing IoT enabled solutions. It is projected that around 500 billion [3] so-called 'smart things' will become part of our day-to-day activities by 2020. Consequently, the IoT faces the challenge of becoming heavy on 'things' while struggling on the connectivity frontier.

A quick glance at the frequency allocation charts provided by the regulatory bodies reveals that most of the prime spectrum is already assigned and the margin for accommodating emerging wireless applications (such as IoT) is low. Consequently, it seems natural to think of the spectrum scarcity as a real challenge posed due to the high utilization of the Hertzian medium. However, a reality check on the usage patterns of the available spectral resources reveals that in a nutshell the spectrum scarcity is nothing but artificial. Spectrum occupancy measurements $[4,5]$ have revealed that these licensed bands are highly under-utilized across space and time. From $13 \%$ to $87 \%$ of the radio spectrum remains unused across spatio-temporal domains. This sporadic utilization of scarce electromagnetic spectrum creates an artificial scarcity which in turn poses the inter-connectivity challenge for IoT. Regulatory bodies such as the FCC (in the USA) and Ofcom (in the UK) have already noticed that such under-utilization of the spectrum can be avoided by more flexible and dynamic spectrum access (DSA) mechanisms [6]. Radio spectrum is a multidimensional entity, i.e., frequency is not the only parameter/dimension which characterizes the spectral opportunity. Space, time, transmission power, polarization, medium access and interference all combinely shape the radio environment. The DSA mechanism employs one or more of these parameters to break the shackles of rigidity imposed by the command and control mechanism. Cognitive radios (CRs) are envisioned to be the key enablers for provisioning DSA. CRs are based on opportunistic exploitation of radio spectrum across one or more dimensions. Nevertheless, while the CR platform renders itself as a promising solution for improving connectivity, its suitability in the context of IoT is limited for two main reasons:

1. High cost: CRs employ sophisticated hardware to derive operational environment awareness and so naturally the radio platforms costs are higher as compared to dumb radio terminals. For IoT solutions, the radio platforms will be embedded inside objects requiring both additional cost and form factors. Thus the radio platforms should be as simple as possible, ideally comprising of a single chip on which a radio transceiver is integrated with the micro-controller unit (MCU). Manufacturers such as Texas Instrument, Nordic Semiconductor, Maxim, CSR, etc., are already providing such simple solutions.

2. Energy consumption and life-time: CR terminals often pay the cost of opportunism in terms of their higher energy consumption. More specifically, the operational environment awareness is driven from the inference process which consumes more energy as compared to simple radio platforms. For the wireless access applications, energy consumption is not considered as a design constraint due to the constant supply of power from the grid. Nevertheless, for IoT based applications energy-consumption is of the utmost important. As discussed earlier, the radio platform is part of a variety of objects, most of them having no/limited access to the power running on coin cell batteries, etc. In this context, the cost of opportunism may be incurred in terms of the reduced operational life-time of these objects.

While object life-time is a critical aspect of design, the issue of so called 'green design' is further brought into play due to the predicted high volume of 'smart things'. Specifically, as predicted in a recent report by Ericsson [3], the $\mathrm{CO}_{2}$ emissions due to a growing number of internet connected devices will increase from 800 Mtonnes to 1200 Mtonnes by 2020. In terms of net emissions, ICT will continue to maintain its $2 \%$ contribution to the global carbon foot-print. Nevertheless, according to the Intergovernmental Panel on Climate Change (IPCC), current emission trends are far from sustainable, requiring an exponential reduction to meet a $2{ }^{\circ} \mathrm{C}$ rise in global temperature. In a recent survey by Cable News Network (CNN) it was estimated that a $2{ }^{\circ} \mathrm{C}$ rise in global temperature will result in a 100 billion US dollar expense rise for addressing various challenges due to climate change. In summary, like all other sectors, ICT should exponentially reduce its energy consumption to operate in an eco-friendly manner. Thus in summary, for future deployment of 500 billion IoT devices a clean 


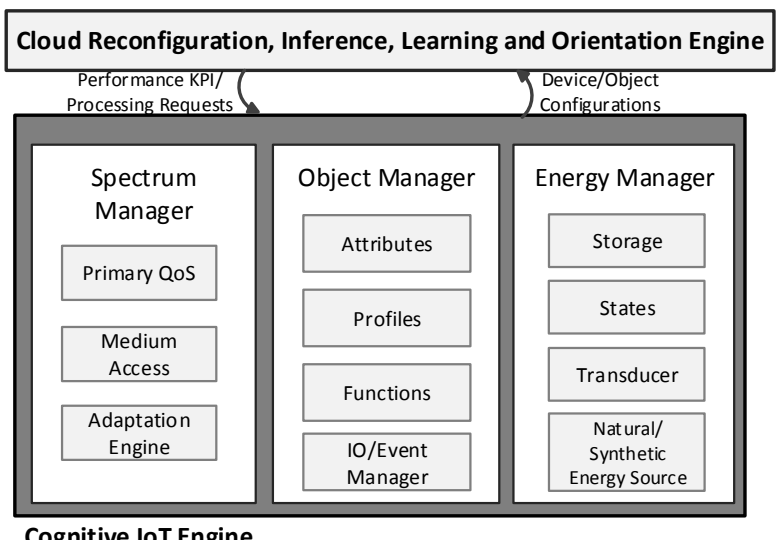

Cognitive loT Engine

Fig. 1 Proposed Architecture for the Cognitive IoT Networks

slate design is necessary to address both energy and spectral efficiency issues.

\subsection{Design Attributes and Proposed Architecture}

The grand challenges posed in the context of the cognitive IoT (CIoT) can be easily translated into design attributes/constraints. To summarize, the radio platform employed in CIoT devices should be: (i) simple yet agile; (ii) spectrally efficient and (iii) low power with a minituarized form factor. To satisfy these design attributes, the definition of cognition in the context of the IoT must be revisited. In particular, not only is spectral agility of a prime importance, but power consumption awareness should also be embedded into the cognitive engine. We advocate that the cognitive engine must be equipped with a potential to harvest energy from ambient sources and in some cases from the objects themselves. For instance, consider smart door locks installed in modern houses. The radio transceivers on these locks can be powered using solar panels harvesting indoor ambient light from both natural and synthetic sources. Moreover, these locks can also harvest power from the mechanical motion of door itself. As smart objects have a very low-duty cycle, harvested energy provides a significant potential for designing self-sustainable so called 'zero-energy consumption' CIoT networks.

In this paper, we propose a cloud enabled CIoT platform as depicted in Fig. 1 to address the aforementioned challenges. From an object oriented programming approach it is well known that an object can be adequately described by its attributes and functionalites. These functionalites and attributes can be linked to external stimuli characterizing events. The behavior of the object in response to an external stimulus is defined by the device profile. External and internal stimului may trigger interrupts which should be handled in accordance with device profile and current state. We propose that this object related functionality should be implemented in the so called 'object manager' which forms the central part of CIoT engine. The object engine coordinates with both the energy and spectrum managers to provide context awareness and indicate required qualityof-service (QoS) or quality-of-information (QoI) constraints. The object management life cycle can be simplified as most of the inference can be moved up to the centralized cloud processor. Thus objects can be made simpler by implementing basic look-up tables which map events, stimulus, attributes and functionality. Notice that the cloud based architecture provides flexibility of re-configuring the object management engine on the fly.

Spectrum and energy management engines are responsible for maximizing the spectral and energy efficiencies of a CIoT network. Unlike data-intensive applications such as cellular networks, where optimizing area spectral efficiency and load balancing are the critical tasks [7], the main purpose of an IoT network is to provide reliable interconnections between smart things. A number of sensors and devices need to communicate with a central controller for inferences, decisions and processing. We advocate the use of a cognitive underlay based spectrum access which requires only transmit power/medium access probability adaptation at the CIoT platforms. The interested reader is refered to [8] for a detailed discussion on exploitation of different degrees of freedom in cognitive underlay networks. In the case of a cognitive underlay mode of operation, it is important to know the spectrum availability and the probability of successful reception when the spectrum is utilized. Moreover, catering for the energy demands of the increased number of CIoT nodes is yet another important issue. The intrinsic advantage of the proposed spectrum access is that its implementation is simple and does not require additional sophisticated hardware. Based on the dynamics of the primary network, the cloud reconfiguration engine can reconfigure the spectrum access probability and the transmit power to guarantee that the QoS of the legacy network is not violated. This is to ensure a robust co-existence framework between the primary users (such as mobile users in cellular network) and the CIoT devices. The practical implementation of such a spectrum access would require a simple look up table at each device so that CIoT platforms do not lose their cost-effectiveness or the form factor by implementing the proposed cognitive access strategy. In the subsequent discussion, we develop an analytical framework for quantifying the performance 
of the large scale CIoT network by considering a reference scenario under the proposed architecture.

\subsection{Outline}

The outline of the rest of the paper is as follows: In section 2, we introduce our reference scenario and mathematical preliminaries which are employed throughout the remainder of this paper. Additionally, we introduce the proposed energy harvesting model and detail its dynamics. In section 3, we define two CIoT performance metrics called (i) the energy success probability and (ii) spectral success probability for the considered CIoT network. We consider a realistic model to compute the harvested power and develop a stochastic model for the energy success probability which can be treated as a proxy for self-sustainability of a CIoT interconnect. Considering, the proposed spectrum management engine, the co-existence constraint is enforced on a CIoT network to provision spectrum access in underlay mode. The co-existence constraint is defined in terms of the average outage probability for an arbitrary mobile user (MU) scheduled in downlink ${ }^{1}$ of the primary network. Notice that in context of the considered primary network, i.e., cellular network, the outage probability is the complement of the coverage probability. Moreover, the averaging is performed over the location of the MU since it is unknown a priori (see [9]). Consequently, the definition of outage for the primary network differs from the one employed in [8]. With the outage analysis of the primary network, we obtain the maximum allowable spectrum access probability for the considered CIoT network under Slotted-ALOHA type protocol $^{2}$. The maximum allowable access probability ensures that the primary user's enforced co-existence constraint is not violated. Section 4 combines the aforementioned performance metrics and introduces a new metric called the overall success probability. The introduced metric provides interesting insight for optimizing the transmit power employed by CIoT nodes to strike a balance between the spectrum access and the energy usage. Section 5 concludes the paper and summarizes the important future directions.

\footnotetext{
1 Notice that the analysis is general and is not affected by considering the uplink of the primary cellular network.

2 In this paper, we consider the Slotted-ALOHA type access strategy for a CIoT network. However, the spectral access probability computed here, can be effectively mapped to the carrier sensing threshold for a CSMA/CA type protocol.
}

\subsection{Notations}

Throughout this paper, we use the following mathematical notations. The probability density of a random variable $X$ is represented by $f_{X}(x)$ and the corresponding cumulative distribution function is denoted by $F_{X}(x)$, where the lowercase letter $x$ is a particular realization of $X$. The expectation of a function with respect to $X$ is represented by $\mathbb{E}_{\mathbb{X}}[$.$] . A bold face lower-case letter,$ for e.g. $\mathbf{y}$, is used to denote a vector on $\mathbb{R}^{2}$ and it's Euclidean norm is represented by $\|\mathbf{y}\|$. Symbol $\backslash$ refers to the exclusion of elements from a set, for instance, $[1,2,3] \backslash[1]=[2,3]$. The expression $b(\boldsymbol{o}, r)$ represents a ball of radius $r$ centered at the origin.

\section{System Model for the Considered Reference Scenario}

In this paper, we consider a large scale energy harvesting CIoT network co-existing with a primary cellular network as shown in Fig. 2. We consider that the CIoT nodes are furnished with solar panels to harvest ambient energy. The CIoT devices with sufficient harvested energy, employ the cognitive underlay approach and utilize the same resource blocks as the primary base stations (BSs) to communicate with the central CIoT controller. We assume that full frequency reuse is employed in the primary cellular network for maximizing the area spectral efficiency. The cognition employed at IoT platforms shapes transmission parameters such that the aggregate interference at the primary MU remains below a certain threshold to satisfy the desired QoS requirement. Consequently, only a fraction of CIoT devices are activated during each transmission sub-frame. From the context of the CIoT network, the accumulation of interference from the primary BSs and other CIoT devices plays a critical role in determining whether the transmissions can be successfully decoded at the corresponding controller. Thus both the opportunity for the transmission and the probability of success when provided with such opportunity collectively define the performance of the CIoT network for the considered reference scenario.

\subsection{Spatial Model}

The spatial distribution of the primary BSs, CIoT nodes and controllers is captured by the independent homogenous Poisson point processes (HPPPs) $\Pi_{p}\left(\lambda_{p}\right), \Pi_{c}\left(\lambda_{c}\right)$ and $\Pi_{k}\left(\lambda_{k}\right)$ respectively, with $\lambda_{i}, i \in[p, c, k]$ being the average number of these entities in a unit area. Mathe- 


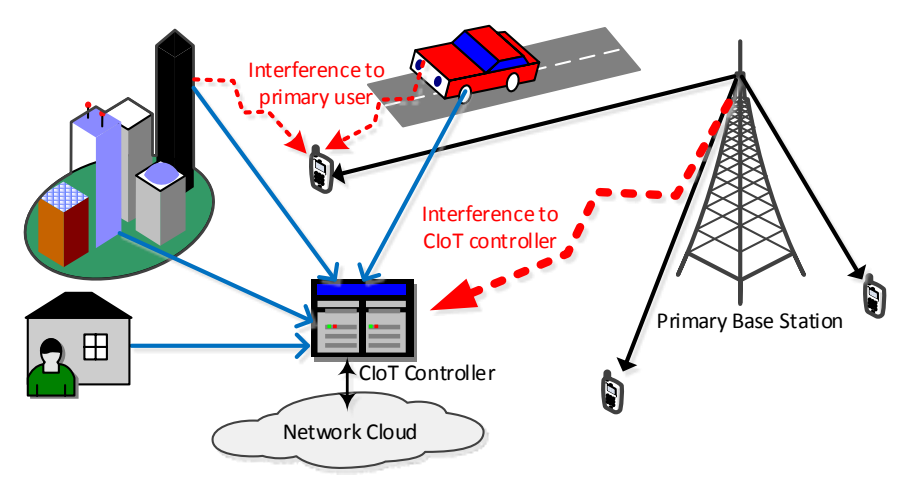

Fig. 2 Top-level diagram showing the coexistence of a CIoT network with the primary cellular network in spectrum underlay mode.

matically, the probability of having $n$ devices in a region $\mathcal{B} \in \mathbb{R}^{2}$ follows the Poisson distribution and is given as

$$
\begin{aligned}
\mathbb{P}\left\{\Pi_{i}(\nu(\mathcal{B}))=n\right\}=\frac{\left(\nu(\mathcal{B}) \lambda_{i}\right)^{n}}{n !} \exp ( & \left.-\nu(\mathcal{B}) \lambda_{i}\right), \\
i & \in[p, m, c, k] .
\end{aligned}
$$

where $\nu(\mathcal{B})=\int_{\mathcal{B}} d \mathbf{x}$ is the Lebesgue measure on $\mathbb{R}^{2}$. For a circular disc of radius $r$ centered at orig (i.e., $\mathcal{B}=$ $b(\boldsymbol{o}, r))$, the Lebesgue measure simplifies to $\nu(\mathcal{B})=\pi r^{2}$ which is simply the area of the disc.

The selection of HPPPs to model the location of the primary BSs and MUs is widely studied in the literature [9]. In the context of CIoTs, the HPPP assumption is quite reasonable as the objects are spatially distributed across the neighborhood with considerable irregularity. Fig. 3 depicts a realization of the network under these considerations. The primary BSs, CIoT devices and controllers are distributed according to a HPPP. The primary MUs (not shown in the figure), as we average the performance metric over all possible locations of MU in each cell. Each MU associates itself with the nearest primary BS. The coverage areas of BSs and CIoT controllers result in separate Voronoi tessellations on $\mathbb{R}^{2}[10]$. Without any loss of generality a probe CIoT controller can be placed at the origin ${ }^{3}$ and the signal from the nearest active CIoT device is considered as the intended signal. All the other active CIoT devices and BSs are considered as interferers for the transmission received on the probe CIoT controller.

\footnotetext{
3 This follows from the Slivnyak's theorem and the palm distribution of HPPPs [11].
}

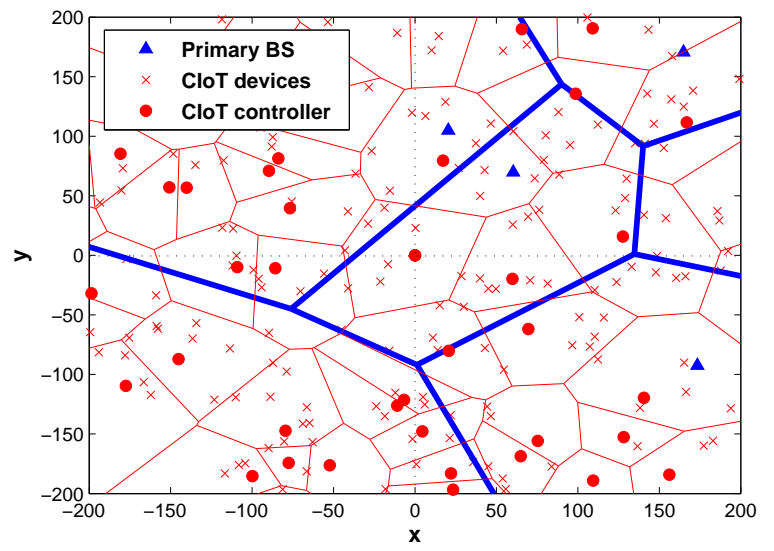

Fig. 3 Realization of the CIoT network in the underlay mode with $\lambda_{p}=10^{-4}, \lambda_{c}=10^{-3}, \lambda_{k}=3 \times 10^{-4}$.

\subsection{Spectrum Access Strategy}

It is assumed that, the CIoT nodes employ random access strategy similar to the Slotted ALOHA MAC protocol to schedule their transmissions over a shared medium for communicating with the corresponding controllers. More specifically, at an arbitrary time instant, the CIoT devices can be classified into two distinct groups, i.e., nodes which are granted spectrum access and those whose transmissions are deferred. If $p_{c}$ denotes the spectrum access probability (SAP) for an arbitrary device $\boldsymbol{x} \in \Pi_{c}{ }^{4}$, then the set of active users un-

4 With a slight abuse of notation, $\boldsymbol{x} \in \mathbb{R}^{2}$ is employed to refer to the node's location as well as the node itself. 
der the considered spectrum access paradigm also forms a HPPP $\Pi_{c}^{\{T X\}}=\left\{\boldsymbol{x} \in \Pi_{c}: \mathbb{1}(\boldsymbol{x})=1\right\}$ with density $\lambda_{c} p_{c}$, where $\mathbb{1}(\boldsymbol{x})$ denotes a Bernoulli random variable and is independent of $\Pi_{c}$.

\subsection{Channel Model}

Throughout this paper, all wireless communication links assume Rayleigh flat fading. We adopt a standard power loss propagation model with the environment dependent path loss exponent $2 \leq \alpha \leq 5$. So the overall channel gain is represented as $h r^{-\alpha}$, where $h \sim \mathcal{E}(1)$ follows a unit-mean exponential distribution representing the received power gain under Rayleigh fading and $r$ is the distance between the transmitter and the receiver. We focus on the interference limited scenario and assume that the effects of the thermal noise on the network's performance are negligible.

\subsection{Energy harvesting}

Harvesting energy from natural (solar, wind, vibration, etc.) and synthesized (microwave power transfer) sources is envisioned as a key enabler for realizing green wireless networks. The choice of a suitable renewable source depends on a number of factors including, the availability of a particular source, ease of harvesting from it and most importantly the energy demand of the application. A detailed comparison of the power densities from various sources is provided in [12]. As solar energy is currently the most scalable renewable resource, from powering small indoor sensors to huge buildings, it naturally becomes a suitable candidate to cater for the contrasting power requirements of things operating under the umbrella of a CIoT. Consequently, in the considered reference scenario, we assume that CIoT nodes are furnished with solar panels to harvest ambient energy. We begin our discussion on the solar energy harvesting in a outdoor setting ${ }^{5}$.

\subsubsection{Solar Energy Harvesting}

The solar power density measured just above the earth's atmosphere is about $1367 \mathrm{Watts} / \mathrm{m}^{2}$ and it is commonly referred to as extra-terrestrial (ET) irradiance. The power density at the surface of the solar panel, also known as insolation, is much less than the ET irradiance and it

\footnotetext{
5 Notice that the model remains same for the indoor setting except for the fact that the output power is attenuated by a factor of 10-100. This is because, indoor panels cannot harvest the direct component of a solar energy field and must rely on the diffuse component.
}

\begin{tabular}{|c|c|}
\hline Hourly okta & Distribution of cloud cover, $f_{k_{c}}()$. \\
\hline \hline 0 & $\mathcal{N}(0.99,0.08)$ \\
\hline$\leq 6$ & $\mathcal{N}(0.6784,0.2046)$ \\
\hline 7 & $\mathcal{W}(0.5577,2.4061)$ \\
\hline 8 & $\mathcal{G}(3.5624,0.0867)$ \\
\hline
\end{tabular}

Table 1 Distribution of clearness index for various levels of cloud cover

depends on a number of geometrical and astronomical aspects such as the earth's elliptical orbit around the sun, earth's tilted axis of rotation, sun's position from directly overhead the panel, the panel's location and its angle of tilt ${ }^{6}$. The aforementioned factors are deterministic and can be quantified. However, the effect of both cloud cover and atmosphere induces randomness in the observed insolation energy field.

While the atmospheric profile of temperature, concentration of water vapor, ozone, aerosol gases, other particles and the surface albedo remains fairly constant during the day, the cloud cover may fluctuate considerably. Meteorologists classify the cloud density in terms of oktas, which are increasing levels of cloud cover with values from $1-8$. The extreme scenarios of the absence of clouds on a clear day and completely overcast conditions or night time are represented by okta 0 and okta 9 respectively. For a particular value of okta or a range of oktas, the atmospheric transmission of the solar energy is commonly parametrized by the clearness index $\left(k_{c}\right)$, which takes the form

$k_{c}=\frac{I}{I_{c}}$,

where, $I_{c}$ is the theoretical clear-sky (cloud free) insolation and $I$ is the instantaneous insolation observed at the panel. This clearness index is a random variable encapsulating the effect of atmospheric variations. The authors in [13] obtained the distributions for $k_{c}$ for a range of oktas as described in Table 1 . They use the data provided by the UK Met Office Integrated Archive System (MIDAS) for hourly values of $I$, and the corresponding cloud okta for the year 2012. For the computation of realistic values of $I_{c}$, they used the DISTORT radiative transfer solver [14] in the libRadtran package [15] with the actual data for the atmospheric conditions. Maximum likelihood estimation (MLE) is used to fit the distributions. To this end, we employ these distributions in our solar panel model to observe the effect of the cloud intensity on the harvested energy.

6 For medium to average size cities, variations in longitude and latitude are not significant. Thus neglecting the environmental randomness, the ET irradiance does not vary significantly over the spatial scale of neighborhood. 


\section{Performance Analysis of an Energy Harvesting Empowered CIoT Network}

Before moving on to the mathematical analysis of the performance determining variables, we define the two key metrics as follows:

Definition 1 Assuming that the harvested energy is utilized for scheduling the transmissions, we define 'energy success probability' as a metric to measure the availability of energy for a certain desired transmit power $P_{c}$. The dynamics of the energy harvester and thus management engine of a CIoT platform can be completely characterized in terms of energy success probability given as [16]

$\epsilon_{\text {suc }}^{\{e\}}=\mathbb{P}\left\{P_{P V} \geq P_{c}\right\}$.

Notice that the definition can be easily modified to cater for the case where energy storage of finite size is present. However, in this article, we do not consider storage and thus harvested energy is employed for transmission scheduling in an instantaneous manner. Such consideration results in a lower bound on actual performance which can be attained by exploiting the energy storage. Furthermore, we assume that transmit energy is the dominant factor in terms of energy consumption and that the power consumption foot-print of the transceiver circuitry is negligible. The circuit power consumption can be accommodated by performing an affine transformation on $P_{c}$.

Definition 2 We define the term 'spectrum success probability' as the probability that a CIoT object is able to access the spectrum and subsequently successfully communicate with the CIoT controller while satisfying the MU's desired QoS constraint. It does this by employing the maximum $\mathrm{SAP} p_{c}^{\max }$.

$\mathbb{P}_{\text {suc }}^{\{c\}}\left(p_{c}^{\max }\right)=\mathbb{E}_{R_{c}}\left[\mathbb{P}\left\{\Gamma_{c}\left(p_{c}^{\max }, r\right) \geq \gamma_{c}\right\}\right]$,

where $\Gamma_{c}$ is the SIR received at a CIoT controller placed at the origin, $R_{c}$ is the random distance between a CIoT device and its nearest controller and $\gamma_{c}$ is the modulation dependent decoding threshold which is selected to satisfy a certain desired frame error rate.

\subsection{Energy Success Probability in Harvesting Empowered CIoT}

We calculate the harvested power using a well-known single diode model for a PV module. The output power can be expressed in terms of the clearness index as follows [17]

$I_{P V}=I_{s c}\left[1-\kappa_{3}\left\{\exp \left(\frac{V_{P V}}{\kappa_{4} V_{o c}}\right)-1\right\}\right]$, where $\kappa_{3}=\left(1-\frac{I_{M P P}}{I_{s c}}\right) \exp \left(\frac{V_{M P P}}{\kappa_{4} V_{o c}}\right)$ and $\kappa_{4}=\left(\frac{V_{M P P}}{V_{o c}}-1\right) / \ln \left(1-\frac{I_{M P P}}{I_{s c}}\right)$. The current generated by the module depends on several parameters: (i) short circuit current $I_{s c}$; (ii) open circuit voltage $V_{o c}$; (iii) maximum power point voltage $V_{M P P}$ and (iv) maximum power point current $I_{M P P}$. These parameters can be expressed as functions of ambient temperature and global horizontal irradiance as follows

$I_{s c}=I_{s c s} \times k_{c} \times\left[1+\varsigma_{1}\left(T-T_{s}\right)\right]$,

$V_{o c}=V_{o c s}+\varsigma_{2}\left(T-T_{s}\right)$,

$I_{M P P}=I_{M P P S} \times k_{c} \times\left[1+\varsigma_{1}\left(T-T_{s}\right)\right]$,

$V_{M P P}=V_{M P P S}+\varsigma_{2}\left(T-T_{s}\right)$,

where $I_{s c s}, V_{o c s}, I_{M P P S}$, and $V_{M P P S}$ are defined for standard conditions, $T_{s}=25^{\circ} \mathrm{C}$ with $\varsigma_{1}$ and $\varsigma_{2}$ being the current and the voltage coefficients. These parameters are generally provided in the data sheet of a PV module. From (5), the output power of the PV panel can be computed as the function of voltage as $P_{P V}=I_{P V} V_{P V}$. For the ease of tractability, we consider standard conditions $\left(T=T_{s}\right.$ ) for the panel for our further analysis. The equation for the harvested power (in Watts) simplifies to

$P_{P V}=k_{c} V_{P V} I_{s c s}\left[1-\kappa_{3}\left\{\exp \left(\frac{V_{P V}}{\kappa_{4} V_{o c s}}\right)-1\right\}\right]$

Using definition 1 and the expression for the output panel power available for transmission, the energy success probability is given as

$\epsilon_{\text {suc }}^{\{e\}}\left(P_{c}\right)=\mathbb{P}\left\{k_{c} \geq \frac{P_{c}}{\tau}\right\}$,

where $\tau=V_{P V} I_{s c s}\left[1-\kappa_{3}\left\{\exp \left(\frac{V_{P V}}{\kappa_{4} V_{o c s}}\right)-1\right\}\right]$.

It is evident from (11) that $\epsilon_{\text {suc }}^{\{e\}}\left(P_{c}\right)$ follows the same distribution as $k_{c}$, only scaled by a factor of $\tau$. The expressions for $\epsilon_{\text {suc }}^{\{e\}}\left(P_{c}\right)$ for various oktas are presented in Table 2. $\mu_{i}$ and $\sigma_{i}, i \in[1,2]$ are the mean and standard deviation of the normal distribution of $k_{c}$ for okta $\in$ $[0-6], \omega$ and $\psi$ are the shape and scale parameters of the Weibull distribution of $k_{c}$ for okta $7, K$ and $\theta$ are the shape and scale parameters of the Gamma distribution of $k_{c}$ for okta 8 and $\gamma(a, x)=\int_{0}^{x} t^{a-1} \exp (-t) d t$ is the lower incomplete Gamma function. For the application of CIoT devices, we consider a mini portable $1.6 \mathrm{~W}$ solar panel which can be used outdoors to power small devices such as sensors, chargers, etc. [18]. 


\begin{tabular}{|c|c|}
\hline Hourly okta & Energy success probability $\left[\epsilon_{s u c}^{\{e\}}\left(\frac{P_{c}}{\tau}\right)=1-F_{k_{c}}\left(\frac{P_{c}}{\tau}\right)\right]$ \\
\hline \hline 0 & $\frac{1}{2}\left[1-\operatorname{erf}\left(\frac{P_{c}-\tau \mu_{1}}{\sqrt{2} \tau \sigma_{1}}\right)\right], \mu_{1}=0.99, \sigma_{1}=0.08$. \\
\hline $1 \leq 6$ & $\frac{1}{2}\left[1-\operatorname{erf}\left(\frac{P_{c}-\tau \mu_{2}}{\sqrt{2} \tau \sigma_{2}}\right)\right], \mu_{2}=0.6784, \sigma_{2}=0.2046$. \\
\hline 7 & $\exp \left(-\left(\frac{P_{c}}{\tau \psi}\right)^{\omega}\right), \omega=2.4061, \psi=0.5577$. \\
\hline 8 & $1-\frac{\gamma\left(K, \frac{P_{c}}{\tau \theta}\right)}{\Gamma(K)}, K=3.5624, \theta=0.0867$. \\
\hline
\end{tabular}

Table 2 Energy success probability for various levels of cloud cover in oktas.

\subsection{Spectrum Access Success Probability in CIoT}

To compute the spectrum success probability, we first consider the outage constraint enforced by the primary network on the CIoT transmitters. The received SIR of a typical MU in an interference limited scenario is characterized as

$$
\begin{aligned}
\Gamma_{p}(r) & =\frac{h_{p} l(\|\boldsymbol{x}\|)}{\sum_{i \in L} h_{i} l\left(\left\|\boldsymbol{x}_{i}\right\|\right)+\sum_{j \in M} \eta g_{j} l\left(\left\|\boldsymbol{x}_{j}\right\|\right)}, \\
& =\frac{h_{p} l(r)}{I_{p}(r)+\eta I_{c}}=\frac{h_{p} l(r)}{I_{\text {tot }}},
\end{aligned}
$$

where $L=\Pi_{p} \backslash b(o,\|\boldsymbol{x}\|), M=\Pi_{c}^{\{T X\}}$ and $h_{p}, h_{i}, g_{j} \sim$ $\mathcal{E}(1)$ are random variables capturing the effect of Rayleigh fading; $l(r)=r^{-\alpha}$ is the path-loss function; $\eta=\frac{P_{c}}{P_{p}}$ is the transmit power ratio of the CIoT and primary networks and $r=\|\boldsymbol{x}\|$ is the distance between the primary BS and the MU. The primary user's QoS constraint can be expressed in terms of its desired SIR threshold $\gamma_{p}$ and an outage probability threshold $\rho_{\text {out }}^{\{p\}}$ as

$\mathbb{P}_{\text {out }}^{\{p\}}\left(P_{c}, p_{c}\right)=\mathbb{E}_{R_{p}}\left[\mathbb{P}\left\{\Gamma_{p}(r) \leq \gamma_{p}\right\}\right] \leq \rho_{\text {out }}^{\{p\}}$,

where $R_{p}$ is the random distance between the MU and its corresponding BS in a reference Voronoi cell. Notice that the primary user's outage probability is coupled with the aggregate interference generated by the CIoT network. Consequently, secondary access is limited subject to the constraint $\rho_{\text {out }}^{\{p\}}$ in (13).

Proposition 1 (Maximum permissible SAP for a CIo $T$ device.) Given the QoS of a MU in terms of the desired SIR threshold $\gamma_{p}$ and its maximum tolerable link outage $\rho_{\text {out }}^{\{p\}}$, the SAP for the CIoT devices which can operate in a concurrent manner without violating the co-existence constraint can be quantified as

$p_{c}^{\max }=\lambda_{p}\left(\frac{1-\left(1-\rho_{\text {out }}^{\{p\}}\right)\left(1+\beta\left(\gamma_{p}, \delta\right)\right)}{\lambda_{c} m \eta^{\delta}\left(1-\rho_{\text {out }}^{\{p\}}\right)}\right)$.

where $m=\Gamma(1+\delta) \Gamma(1-\delta), \delta=2 / \alpha$ and $\beta\left(\gamma_{p}, \delta\right)=$ $\frac{\delta \gamma_{p}}{1-\delta}{ }_{2} F_{1}\left(1,1-\delta ; 2-\delta ;-\gamma_{p}\right)$. Here ${ }_{2} F_{1}(a, b ; c ; z)$ is the Gauss Hypergeometric function [19]. For $\alpha=4(\delta=$ $1 / 2), \beta\left(\gamma_{p}, \delta\right)=\sqrt{\gamma_{p}} \arctan \left(\sqrt{\gamma_{p}}\right)$ and the maximum permissible $S A P$ simplifies to $p_{c}^{\max }=\lambda_{p}\left(\frac{1-\left(1-\rho_{\text {out }}^{\{p\}}\right)\left(1+\sqrt{\gamma_{p}} \arctan \left(\sqrt{\gamma_{p}}\right)\right)}{\lambda_{c} m \sqrt{\eta}\left(1-\rho_{\text {out }}^{\{p\}}\right)}\right)$,

Proof Following the steps in [9] and [8], the outage probability of the primary MU can be written as

$$
\mathbb{P}_{\text {out }}^{\{p\}}\left(P_{c}, p_{c}\right)=1-
$$

For the considered scenario, $\quad \mathcal{L}_{I_{\text {tot }}}(s)=\mathcal{L}_{I_{p}(r)}(s) \times$ $\left.\mathcal{L}_{I_{c}}(\eta s)\right|_{s=\gamma_{p} r^{\alpha}}{ }^{7}$, and $\mathcal{L}_{I_{p}(r)}(s)$ and $\mathcal{L}_{I_{c}}(\eta s)$ are respectively the Laplace transforms of the interference on the aggregate interference on the MU from the other-cell co-channel interferers and the spectrum sharing CIoT devices. Using the well-known definition of the generating functional of a HPPP in [11], the following Laplace transforms can be evaluated as

$$
\begin{aligned}
\mathcal{L}_{I_{p}}\left(\gamma_{p} r^{\alpha}\right)= & \exp \left\{-2 \pi \lambda_{p}\right. \\
& \left.\int_{r}^{\infty}\left(1-\mathbb{E}_{h}\left[\exp \left(-\gamma_{p} r^{\alpha} h v^{-\alpha}\right)\right]\right) d v\right\} \\
= & \exp \left\{-\pi \lambda_{p} r^{2} \beta\left(\gamma_{p}, \delta\right)\right\}
\end{aligned}
$$

and

$$
\begin{aligned}
\mathcal{L}_{I_{c}}\left(\eta \gamma_{t h}^{\{p\}} r^{\alpha}\right)= & \exp \left\{-2 \pi \lambda_{c}\right. \\
& \left.\int_{0}^{\infty}\left(1-\mathbb{E}_{g}\left[\exp \left(-\gamma_{p} r^{\alpha} g u^{-\alpha}\right)\right]\right) d u\right\} \\
= & \exp \left\{-\pi m \lambda_{c} p_{c} \gamma_{p}^{\delta} \eta^{\delta} r^{2}\right\}
\end{aligned}
$$

where $m=\Gamma(1+\delta) \Gamma(1-\delta), \delta=2 / \alpha$ and $\beta\left(\gamma_{p}, \delta\right)=$ $\gamma_{p}^{\delta} \int_{\gamma_{p}^{-\delta}}^{\infty}\left(1+u^{\frac{1}{\delta}}\right)^{-1} d u$. The solution for this integral in terms of the hypergeometric function can be obtained by substituting $y=u^{\frac{1}{\delta}}$ and using Eq. 3.194-2.6 from [20].

Notice the difference in the limits of integration in (17) and (19). This is due to the fact that the other-cell interferer will be separated by a minimum distance of

7 This is because of the independence of the point processes $\Pi_{p}\left(\lambda_{p}\right)$ and $\Pi_{c}\left(\lambda_{c}\right)$. 
$r$ from the tagged MU while such a constraint is not enforced on the CIoT devices. Inserting the above two expressions into (16) and solving the integral provides us with the outage probability of the MU. Furthermore, enforcing the outage constraint $\rho_{\text {out }}^{\{p\}}$ as in (13) and inverting the equation for $p_{c}$ concludes the proof.

Implementation Note: From (14), we notice that $p_{c}^{\max }$ is function of both the primary and the IoT network parameters. Thus cloud based coordination is benefical to dynamically reconfigure the SAP based on the prevelant network conditions. More specifically, (14) can be implemented in the cloud re-configuration, inference, learning and adaptation engine to provison selforganization.

Proposition 2 (Spectrum success probability of the CIoT. The probability that a CIoT user is able to access the available spectrum and successfully communicate with its controller in the uplink is given as

$\epsilon_{s u c}^{\{s\}}\left(P_{c}\right)=\frac{1}{1+p_{c}^{\max } \beta\left(\gamma_{c}, \delta\right)+\frac{\lambda_{p}}{\lambda_{c}} m \eta^{-\delta} \gamma_{c}}$.

Proof The proof for (21) follows similar steps as in the proof of (16). The received SIR at the CIoT controller placed at the origin is given as

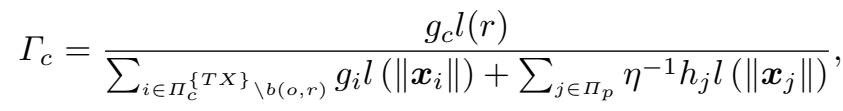

$$
\begin{aligned}
& =\frac{h_{c} l(r)}{\eta^{-1} I_{p}+I_{c}(r)}=\frac{h_{c} r^{-\alpha}}{I_{t o t}} \text {. }
\end{aligned}
$$

Using the definition 4 , the spectrum success probability is given as

$$
\mathbb{P}\left\{\Gamma_{c} \geq \gamma_{c}\right\}=\int_{r>0} \mathcal{L}_{I_{t o t}}\left(\gamma_{c} r^{\alpha}\right) \exp \left(-\pi \lambda_{c} r^{2}\right) 2 \pi \lambda_{c} r d r .
$$

In this case, $\mathcal{L}_{I_{\text {tot }}}(s)$ will be

$\mathcal{L}_{I_{t o t}}(s)=\mathcal{L}_{I_{p}}\left(\eta^{-1} s\right) \times\left.\mathcal{L}_{I_{c}}(s)\right|_{s=\gamma_{c} r^{\alpha}}$,

and the limits of integration are interchanged for the Laplace transform of the primary and the CIoT interferences as now the underlay network is under consideration and the interference is being measured on the CIoT controller in the uplink. Hence, the Laplace transforms are given by the following expressions

$$
\begin{aligned}
\mathcal{L}_{I_{p}}\left(\eta^{-1} \gamma_{c} r^{\alpha}\right)= & \exp \left\{-2 \pi \lambda_{c}\right. \\
& \left.\int_{0}^{\infty}\left(1-\mathbb{E}_{h}\left[\exp \left(-\gamma_{p} r^{\alpha} h v^{-\alpha}\right)\right]\right) d v\right\} \\
= & \exp \left\{-\pi m \lambda_{p} \gamma_{c}^{\delta} \eta^{-\delta} r^{2}\right\}
\end{aligned}
$$

and

$$
\begin{aligned}
\mathcal{L}_{I_{c}}\left(\gamma_{c} r^{\alpha}\right)= & \exp \left\{-2 \pi \lambda_{c}\right. \\
& \left.\int_{r}^{\infty}\left(1-\mathbb{E}_{g}\left[\exp \left(-\gamma_{p} r^{\alpha} g u^{-\alpha}\right)\right]\right) d u\right\} \\
= & \exp \left\{-\pi \lambda_{c} p_{c} \beta\left(\gamma_{c}, \delta\right) r^{2}\right\},
\end{aligned}
$$

where $m=\Gamma(1+\delta) \Gamma(1-\delta), \delta=2 / \alpha$ and $\beta\left(\gamma_{c}, \delta\right)=$ $\gamma_{c}^{\delta} \int_{\gamma_{c}^{-\delta}}^{\infty}\left(1+w^{\frac{1}{\delta}}\right)^{-1} d w=\frac{\delta \gamma_{c}}{1-\delta}{ }_{2} F_{1}\left(1,1-\delta ; 2-\delta ;-\gamma_{c}\right)$.

\section{Overall Success Link Probability}

Employing the existing analytical characterizations, we define the unified performance metric for the energy harvesting empowered CIoT network. Intuitively, a CIoT object will only be able to successfully communicate with its nearest controller if the following conditions are met:

1. The harvested energy is greater than the required transmit power;

2. The object is allowed to access spectrum while satisfying the primary MU's QoS constraint; and

3 . The ratio of the received signal's power to the interference from all the other CIoT devices and the primary BSs is greater than the desired SIR threshold $\gamma_{c}$.

The performance metrics $\epsilon_{\text {suc }}^{\{s\}}\left(P_{c}\right)$ and $\epsilon_{\text {suc }}^{\{e\}}\left(P_{c}\right)$ derived in the previous section are both important in characterizing the performance of a CIoT network. Nevertheless, $\epsilon_{s u c}^{\{e\}}\left(P_{c}\right)$ only signifies the energy availability and $\epsilon_{s u c}^{\{s\}}\left(P_{c}\right)$ signifies the spectrum availability. For a more comprehensive analysis, there is a need to combine these two metrics such that all the factors governing the performance of a CIoT network can be captured by a single metric. Hence, we introduce the overall link success probability metric as follows

$\epsilon_{\text {suc }}^{\text {tot }}\left(P_{c}\right)=\epsilon_{\text {suc }}^{\{s\}}\left(P_{c}\right) \times \epsilon_{\text {suc }}^{\{e\}}\left(P_{c}\right)$,

where $\epsilon_{\text {suc }}^{\text {tot }}\left(P_{c}\right)$ is the overall success probability, which depends on the communication aspects, CIoT transmit power, and the solar panel parameters. Using the expressions in Table 2 and with a few manipulations in (21), the overall success probability in (28) is given as

For okta $\leq 6$ :

$$
\begin{array}{r}
\epsilon_{\text {suc }}^{t o t}\left(P_{c}\right)=\frac{1}{1+\xi P_{c}^{-\delta}} \frac{1}{2}\left[1-\operatorname{erf}\left(\frac{P_{c}-\tau \mu_{i}}{\sqrt{2} \tau \sigma_{i}}\right)\right], \\
i \in[1,2],
\end{array}
$$

For okta 7 :

$$
\epsilon_{\text {suc }}^{\text {tot }}\left(P_{c}\right)=\frac{1}{1+\xi P_{c}^{-\delta}} \exp \left(-\left(\frac{P_{c}}{\tau \psi}\right)^{\omega}\right)
$$


For okta 8 :

$\epsilon_{\text {suc }}^{\text {tot }}\left(P_{c}\right)=\frac{1}{1+\xi P_{c}^{-\delta}}\left[1-\frac{\gamma\left(K, \frac{P_{c}}{\tau \theta}\right)}{\Gamma(K)}\right]$

where $\xi=P_{p}^{\{o p t\}^{\delta}}\left(a_{1}+a_{2}\right) ; a_{1}=m \gamma_{c}^{\delta} \frac{\lambda_{p}}{\lambda_{c}}, a_{2}=\lambda_{p} \beta\left(\gamma_{c}, \delta\right)$ $\times \frac{1-\left(1-\rho_{\text {out }}^{\{p\}}\right)\left(1+\beta\left(\gamma_{p}, \delta\right)\right)}{\left(1-\rho_{\text {out }}^{\{p\}}\right) m \lambda_{c} \gamma_{p}^{\delta}}, i=1$ represents okta 0 , while $i=2$ represents $1 \leq$ okta $\leq 6$.

It follows from (29), (30) and (31) that for all values of oktas, the effect of modulation dependent decoding thresholds $\gamma_{c}$ and $\gamma_{p}$, path loss exponent $\delta$, CIoT device density $\lambda_{c}$ and primary BS density $\lambda_{p}$ remains the same on the overall success probability. This is because the cloud cover only affects the availability of the energy and the spectrum success probability remains unchanged. However, from (28), we observe that both the terms $\epsilon_{\text {suc }}^{\{s\}}\left(P_{c}\right)$ and $\epsilon_{\text {suc }}^{\{e\}}\left(P_{c}\right)$ depend on the device level transmit power $P_{c}$, which could be adapted to optimize the CIoT performance. In the following section, we see how $P_{c}$ could be adapted to achieve better connectivity for the CIoT devices and also, what impact will the densification of CIoTs devices have on the required transmit power and the coverage characteristics.

\subsection{Optimal transmit power}

As the CIoT transmit power $P_{c}$ increases (and in turn $\eta$ increases keeping $P_{p}$ constant), $\epsilon_{s u c}^{\{s\}}\left(P_{c}\right)$ also increases as the maximum permissible $\operatorname{SAP}\left(p_{c}^{\max }\right)$ decreases. This implies that transmission opportunities of CIoT devices will diminish due to interference protection implemented by the cloud controller to guarantee the primary user's QoS requirement. On the contrary, increasing $P_{c}$ results in a drop in $\epsilon_{s u c}^{\{e\}}\left(P_{c}\right)$ as more and more harvested power is required to meet the energy demand. However, the low transmit power employed by a CIoT platform may not be able to guarantee the required QoS or QoI at each CIoT node. Consequently, the transmit power must be optimized through considering energy and spectral success. The inverse relationship between the two terms can be observed in Fig. 4. Since the overall success probability is the product of these two terms, there must exist an optimal power which maximizes the overall success probability. We see in Fig. 5 that as the cloud okta increases and consequently the harvested energy decreases, the overall success probability decreases and for a fixed value of cloud okta, there always exists an optimal transmit power which maximizes the overall success probability.

Proposition 3 (Optimal Transmit Power) The optimal transmit power can be numerically evaluated by solving the following equations for $P_{c}^{\{o p t\}}$ :

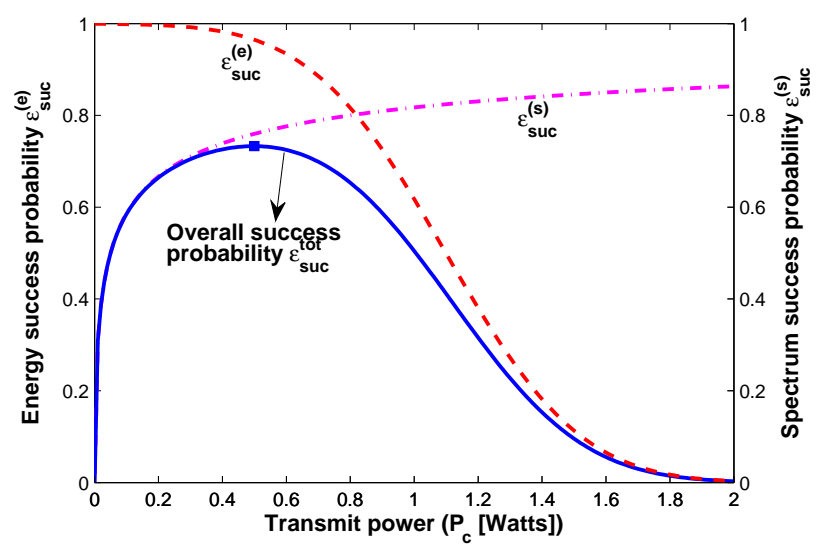

Fig. 4 Existence of an optimal power which maximizes the overall success probability with $1 \leq$ okta $\leq$ $6, \lambda_{c} / \lambda_{p}=10, \alpha=4, \rho_{\text {out }}^{\{p\}}=0.2, P_{p}=10 \mathrm{~W}, \gamma_{c}=$ $-7 d B, \gamma_{p}=-6 d B, I_{s c s}=0.277 A, V_{o c s}=6.9 \mathrm{~V}, V_{M P P S}=$ $6 V, I_{M P P S}=0.27 A$ (see Eq. (11), (21) and (29)).

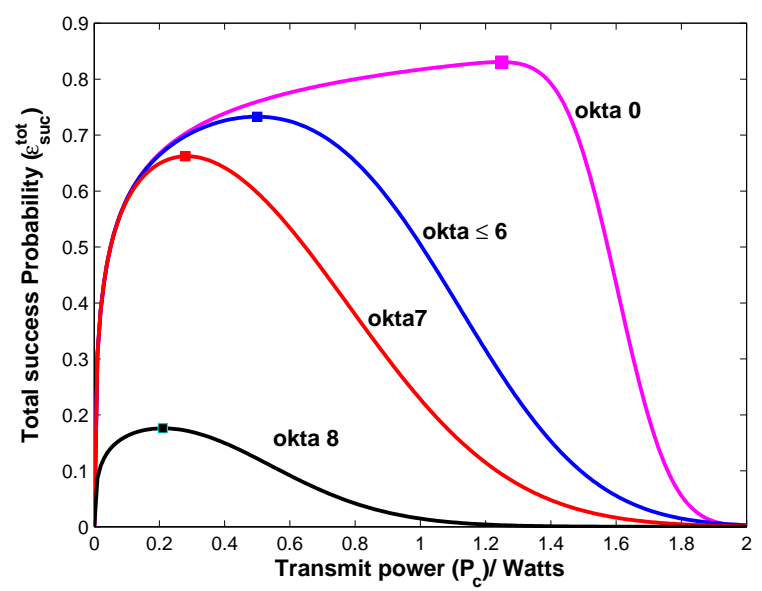

Fig. 5 Overall success probability with transmit power adaptation for various okta values, for $\lambda_{c} / \lambda_{p}=10, \alpha=$ $4, \rho_{\text {out }}^{\{p\}}=0.2, P_{p}=10 \mathrm{~W}, \gamma_{c}=-7 d B, \gamma_{p}=-6 d B, I_{s c s}=$ $0.277 \mathrm{~A}, V_{\text {ocs }}=6.9 \mathrm{~V}, V_{M P P S}=6 \mathrm{~V}, I_{M P P S}=0.27 \mathrm{~A}$ (see Eq. (29)-(31) and (32)-(34)).

For okta $\leq 6$ :

$$
\begin{aligned}
& \sqrt{\frac{2}{\pi}} \frac{P_{c}^{\{o p t\}}}{\tau \sigma_{i}}\left(\xi+P_{c}^{\{o p t\}^{\delta}}\right) \exp \left(-\frac{1}{2}\left(\frac{\tau \mu_{i}-P_{c}^{\{o p t\}}}{\tau \sigma_{i}}\right)^{2}\right) \\
& +\xi \delta\left[\operatorname{erf}\left(\frac{P_{c}^{\{o p t\}}-\tau \mu_{i}}{\sqrt{2} \tau \sigma_{i}}\right)-1\right]=0, i \in[1,2]
\end{aligned}
$$

For okta 7 :

$P_{c}^{\{o p t\}^{\delta+\omega}}+\xi P_{c}^{\{o p t\}^{\omega}}-\frac{\delta \xi(\tau \psi)^{\omega}}{\omega}=0$, 
For okta 8 :

$$
\begin{array}{r}
\left(\frac{P_{c}^{\{o p t\}}}{\tau \theta}\right)^{K} \exp \left(-\frac{P_{c}^{\{o p t\}}}{\tau \theta}\right)\left(\xi+P_{c}^{\{o p t\}^{\delta}}\right)+ \\
\xi \delta \gamma\left(K, \frac{P_{c}^{\{o p t\}}}{\tau \theta}\right)-\xi \delta \Gamma(K)=0 .
\end{array}
$$

Proof The optimal transmit power $\left(P_{c}^{\{o p t\}}\right)$ is the solution of

$\frac{\partial \epsilon_{s u c}^{t o t}\left(P_{c}\right)}{\partial P_{c}}=0$

From (35), we obtain the partial derivatives of $\epsilon_{\text {suc }}^{t o t}$ for various oktas, which can be numerically solved to obtain the optimal transmit power of the CIoT.

\subsubsection{Discussion}

1. As seen from Figs. 4 and 5, there always exists an optimal transmit power point which maximizes the overall success probability. This is due the fact that as the transmit power increases, the maximum SAP $\left(p_{c}^{\max }\right)$ reduces to activate lesser CIoT devices and the SIR increases and hence the probability of successful transmission increases. However, as the desired transmit power goes high, the available harvested energy becomes insufficient. The optimal transmit power point may lie in either the spectrum limited regime, i.e. when $\epsilon_{\text {suc }}^{\{e\}}\left(P_{c}\right)>\epsilon_{\text {suc }}^{\{s\}}\left(P_{c}\right)$ or the energy limited regime, i.e. when $\epsilon_{\text {suc }}^{\{s\}}\left(P_{c}\right)>\epsilon_{\text {suc }}^{\{e\}}\left(P_{c}\right)$. The location of the optimal point depends solely on the solar panel parameters and other network related parameters which affect the slope of $\epsilon_{s u c}^{\{e\}}\left(P_{c}\right)$ and $\epsilon_{\text {suc }}^{\{s\}}\left(P_{c}\right)$. As illustrated in Fig. 4, the decrease in $\epsilon_{\text {suc }}^{\{e\}}\left(P_{c}\right)$ is more rapid than the increase in $\epsilon_{\text {suc }}^{\{s\}}\left(P_{c}\right)$ for the given set of parameters hence, the optimal power point lies in the spectrum limited regime.

2. Cloud cover plays an immensely important role in determining the CIoT performance. Comparing the clear sky (okta 0) and heavily cloudy conditions (okta 8) in Fig. 5, a drop of almost $76 \%$ in the maximum overall success probability is observed while for partially cloudy conditions, this drop is fairly reasonable (around $17 \%$ and $24 \%$ for okta 6 and less and okta 7 respectively). Not only does the cloud cover affect the maximum overall success probability, it also changes the optimal transmit power. This can be intuitively explained by the fact that as the sky becomes clearer, a higher amount of energy can be harvested and also consumed in achieving a high spectral coverage. Thus, the a higher optimal power can be adopted when the harvested energy is high.

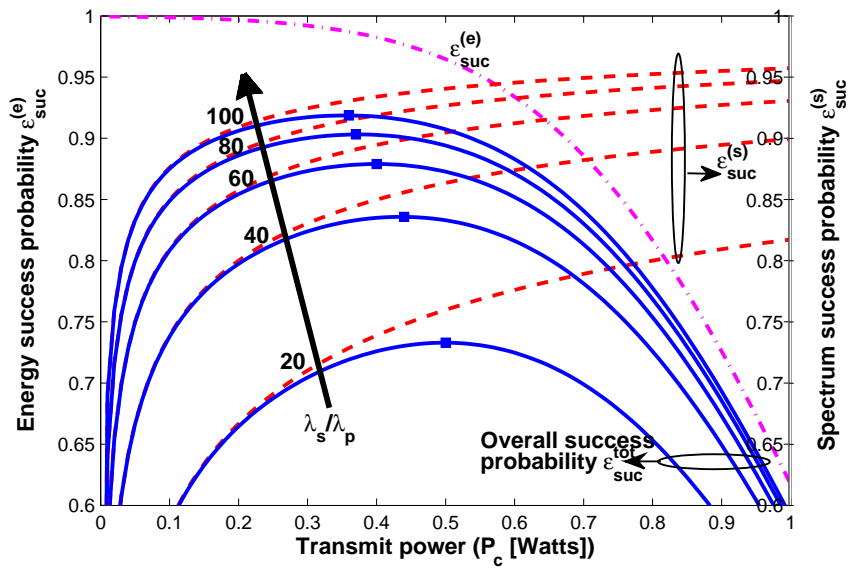

Fig. 6 Effect of changing transmit power on energy success, Spectrum success and overall success probabilities for various values of CIoT density with $1 \leq$ okta $\leq 6, \lambda_{p}=10^{-4}, \alpha=$ $4, \rho_{\text {out }}^{\{p\}}=0.2, P_{p}=10 \mathrm{~W}, \gamma_{c}=-7 \mathrm{~dB}, \gamma_{p}=-6 \mathrm{~dB}, I_{s c s}=$ $0.277 \mathrm{~A}, V_{\text {ocs }}=6.9 \mathrm{~V}, V_{M P P S}=6 \mathrm{~V}, I_{M P P S}=0.27 \mathrm{~A}(\mathrm{see}$ Eq. (11), (21) and (29)).

3. In heavily cloudy conditions (okta 8), optimizing transmit power is of a little or no value as the CIoT devices remain in outage for $80 \%$ of the time because of the lack of harvested solar energy. Hence, it is more effective to turn off the devices completely or switch to a backup energy supply.

4. As the relative density of CIoT devices is increased, the spectrum success probability increases and the overall success probability also increases as observed in Fig. 6. Even though the maximum SAP of CIoTs is inversely related to its density (14), the non-linear increase in overall success is attributed to the fact that the CIoT controller is closer to the device itself. This greatly reduces path loss for the desired signal. Figs. 6 and 7 illustrate the diminishing gains in optimal power with the increase in the CIoT density.

\section{Conclusion}

In this article, we provided a unified architecture for the cognitive internet-of-things (CIoT) framework. We advocate that the definition of cognition must be extended to incorporate IoT specific design challenges. We solicited a cloud based cognitive underlay spectrum access for the IoT radio platforms. Furthermore, energy harvesting is proposed to attain so called self-sustainable network design. We introduce a novel statistical framework to characterize the energy and spectral success in CIoT networks. The relationship between energy and spectral outages was explored for a reference scenario of solar energy harvesting with stochastic cloud cover. 


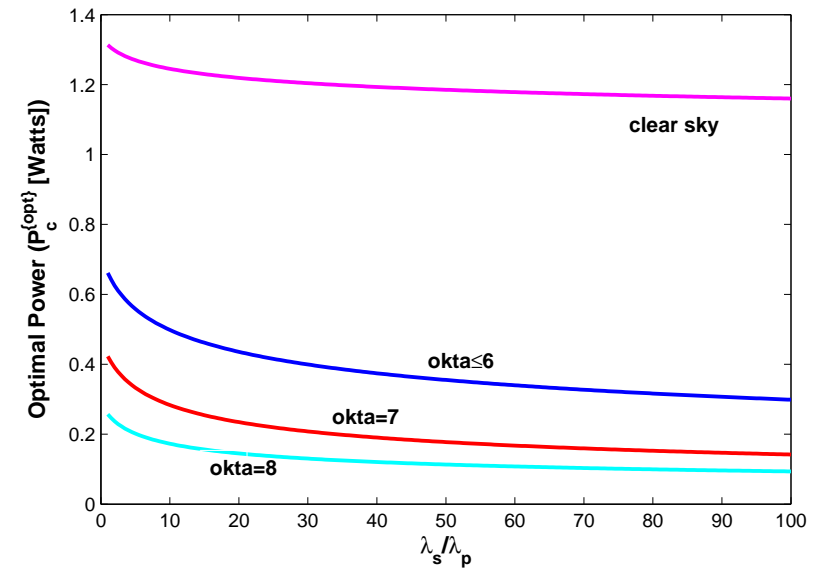

Fig. 7 Effect of changing the CIoT density on the optimal transmit power $P_{c}^{o p t}, \lambda_{p}=10^{-4}, \alpha=4, \rho_{\text {out }}^{\{p\}}=0.2, P_{p}=$ $10 W, \gamma_{c}=-7 d B, \gamma_{p}=-6 d B, I_{s c s}=0.277 A, V_{o c s}=$ $6.9 \mathrm{~V}, V_{M P P S}=6 \mathrm{~V}, I_{M P P S}=0.27 \mathrm{~A}$.

It was shown that both metrics are coupled as 'overall success' as they are governed by same underlying parameter, i.e., transmit power. Finally, there exists a tradeoff between maximizing spectral success and the availability of energy and thus an analytical framework was developed to obtain the optimal transmit power to maximize network level performance.

\section{References}

1. M. Weiser, "The computer for the 21st century," Scientific american 265(3), pp. 94-104, 1991.

2. E. I. Unit, "The internet of things business index," ARM , pp. 1-22, 2014.

3. E. I. Unit, "Ericsson energy and carbon report: on the impact of networked society," Ericsson , pp. 1$11,2013$.

4. M. A. McHenry, P. A. Tenhula, D. McCloskey, D. A. Roberson, and C. S. Hood, "Chicago spectrum occupancy measurements \& analysis and a long-term studies proposal," in Proceedings of the first international workshop on Technology and policy for accessing spectrum, p. 1, ACM, 2006.

5. M. A. McHenry, "Nsf spectrum occupancy measurements project summary," Shared spectrum company report, 2005.

6. E. FCC, "Docket 98-153," First Report and Order: Revision of Part 15, 2002.

7. J. G. Andrews, S. Singh, Q. Ye, X. Lin, and H. S. Dhillon, "An overview of load balancing in hetnets: Old myths and open problems," Wireless Communications, IEEE 21(2), pp. 18-25, 2014.
8. S. A. R. Zaidi, M. Ghogho, and D. C. McLernon, "Breaking the area spectral efficiency wall in cognitive underlay networks," IEEE Journal on Selected Areas in Communications, 2014.

9. J. G. Andrews, F. Baccelli, and R. K. Ganti, "A tractable approach to coverage and rate in cellular networks," Communications, IEEE Transactions on 59(11), pp. 3122-3134, 2011.

10. A. Okabe, B. Boots, K. Sugihara, and S. N. Chiu, Spatial tessellations: concepts and applications of Voronoi diagrams, vol. 501, John Wiley \& Sons, 2009.

11. S. N. Chiu, D. Stoyan, W. S. Kendall, and J. Mecke, Stochastic geometry and its applications, John Wiley \& Sons, 2013.

12. F. Yildiz, "Potential ambient energy-harvesting sources and techniques.," Journal of Technology Studies 35(1), pp. 40-48, 2009.

13. J. Bright, C. Smith, P. Taylor, and R. Crook, "Stochastic generation of minutely irradiance time series derived from mean hourly weather observation data," Solar Energy , 2014.

14. K. Stamnes, S.-C. Tsay, W. Wiscombe, and I. Laszlo, "Disort, a general-purpose fortran program for discrete-ordinate-method radiative transfer in scattering and emitting layered media: documentation of methodology," 2000.

15. B. Mayer, A. Kylling, C. Emde, U. Hamann, and R. Buras, "libradtran users guide," 2011.

16. S. A. R. Zaidi, M. Z. Shakir, M. A. Imran, M. Ghogho, A. Vasilakos, K. Qaraqe, and D. McLernon, "Cognitive internet of things: A unified perspective," International Conference on Software-Defined and Virtualized Future Wireless Networks , 2014.

17. S. A. R. Zaidi, M. Ghogho, D. C. McLernon, and A. Swami, "Energy harvesting empowered cognitive metro-cells," IEEE Workshop on Cognitive Cellular Systems , 2014.

18. "Mini solar panel." http://xrsolar en .alibaba. com/product/1950397751-221804628/1\_6w \ _Mini\_Solar\_Panel.html, 2011.

19. G. Gasper and M. Rahman, Basic hypergeometric series, vol. 96, Cambridge university press, 2004.

20. A. Jeffrey and D. Zwillinger, Table of integrals, series, and products, Academic Press, 2007. 


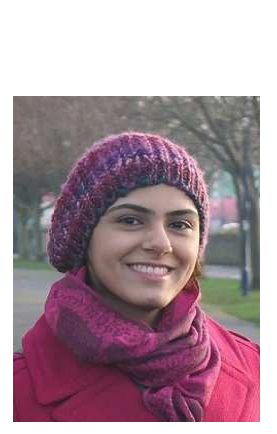

Asma Afzal received the B.E. and M.S. degrees in electrical engineering from $\mathrm{Na}$ tional University of Sciences and Technology (NUST), Islamabad, Pakistan, in 2011 and 2013, respectively. She is currently working toward the Ph.D. degree in electrical engineering from University of Leeds, Leeds, U.K. During her masters, she worked as a Lab Engineer in NUST on a collaborative project with Cypress Semi-Conductor, San Jose, CA, USA. Her research interests lie in stochastic modeling and analysis of future generation wireless networks with energy harvesting.

Syed Ali Raza Zaidi is currently

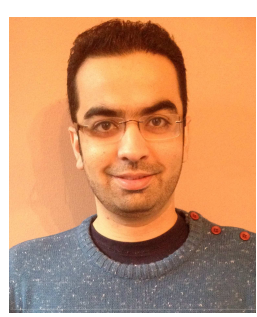

a research fellow at University of Leeds on the US army research lab funded project: 'Cognitive Green Wireless Communication- A Network Science Perspective'. He received his B.Eng degree in information and communication system engineering from the School of Electronics and Electrical Engineering, NUST, Pakistan in 2008 and $\mathrm{PhD}$ from University of Leeds in 2013. He was awarded University

of Leeds' F. W. Carter prize for best PhD thesis. He was also awarded the NUST's most prestigious Rector's gold medal for his final year project. From September 2007 till August 2008, he served as a Research Assistant in Wireless Sensor Network Lab on a collaborative research project between NUST, Pakistan and Ajou University, South Korea. In 2008, he was awarded overseas research student (ORS) scholarship along with Tetley Lupton and Excellence Scholarships to pursue his $\mathrm{PhD}$ at the School of Electronics and Electrical Engineering, University of Leeds, U.K. He was also awarded with COST IC0902, DAAD and Royal Academy of Engineering grants to promote his research. He was a visiting research scientist at Qatar Innovations and Mobility Center from October to December 2013. He has served as workshop chair for IEEE VTC 2015 DCS, IEEE IWCMC 2015 ReAP, CROWNCOM 2015 and IEEE CAMAD 2015 ReAP. He has served as an invited reviewer for IEEE flagship journals and conferences. Over past few years, Dr. Zaidi has served as TPC Member for IEEE WCNC 12-15, IEEE VTC 10-15, EUSIPCO 11-15 and SPAWC 10. IEEE His research is focused towards design and analysis of the large scale ad-hoc wireless networks by employing tools from stochastic geometry and random graph theory.

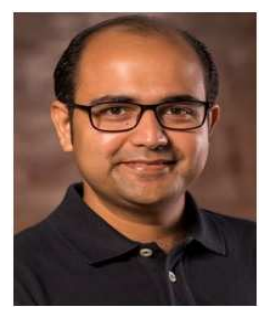

Muhammad Zeeshan Shakir is an Assistant Research Scientist with the Wireless Research Lab, Texas A\&M University at Qatar (TAMUQ), Doha, Qatar, since July 2012. Previously, from November 2009 to June 2012, he was a Research Fellow with the Communication Technology Lab, King Abdullah University of Science and Technology (KAUST), Thuwal, Saudi Arabia. In 2010, he received a Collaborative Research Fund from the KAUST Global Collaborative Research initiative to start bilateral collaboration with foreign universities. From September to December 2010, he was a Visiting Researcher with the Center for Communication System Research (CCSR), University of Surrey, Guildford, UK. In 2010, he earned his PhD degree in electronic and electrical engineering from University of Strathclyde, Glasgow, UK. From January 2006 to September 2009, he was the joint recipient of an industrial research fund and a prestigious overseas research scholarship by the University of Strathclyde and Picsel Technologies Ltd. UK. Dr. Shakir's research interests include design and deployment of hyper-dense heterogeneous small-cell networks, backhaul networking and communications and Green communications. $\mathrm{He}$ is also interested in deriving emerging wireless application oriented unified frameworks for machine type communications, emerging cognitive radio communications and ubiquitous remote/mobile healthcare communications. He has published/produced more than 75 technical journal and conference papers and has contributed to 6 books, all in well reputed venues. His most of the research has been sponsored by Qatar National Research Fund (QNRF) and national industrial partners.

Dr. Shakir has been/is giving tutorials on emerging Green heterogeneous wireless communication systems at IEEE flagship conferences including IEEE PIMRC 2013, London, IEEE ICC 2014, Sydney and IEEE GLOBECOM 2014, Austin. He has been the organizer and technical chair for several international conferences and special sessions/symposium/workshops collocated with major IEEE conferences. He is the organizer and technical chair of debut edition of IEEE Workshop on Next Generation of Backhaul/Fronthaul Networks (BackNets 2015) in conjunction with IEEE ICC 2015. He has been a member of technical program committee of several IEEE flagship conferences, such as the IEEE ICC 2015, IEEE WCNC 2014, and IEEE GLOBECOM 2014. He is an Associate Technical Editor, IEEE Communications Magazine. At present, he is serving as a Lead Guest Editor for a special issue on "Cognitive Cellular Systems" in IEEE Communication Magazine and a feature topic on "Smart Backhauling for 5G" in IEEE Wireless Communications. Currently, he has been serving as an Elected Secretary to the IEEE DySPAN 1900.7 working group and the standard representative of ComSoc technical committee on Signal processing and communication electronics. He is an active member of IEEE and IEEE Standard Association. 


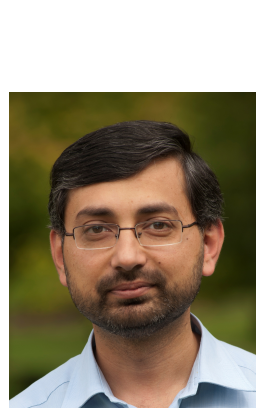

Muhammad Ali Imran Surrey (the 5G innovation Centre and an outdoor cellular test bed is being developed at Surrey University with a recent grant of above $£ 35 \mathrm{~m}$ ). He has a global collaborative research network spanning both academia and key industrial players in the field of wireless communications. $\mathrm{He}$ has supervised 17 successful PhD graduates and published over 150 peer-reviewed research papers including more than 20

IEEE Journals. His research interests include the derivation of information theoretic performance limits, energy efficient design of cellular system and learning/self-organizing techniques for optimization of cellular system operation. He was tutorial chair for EW 2013 and TPC member of several IEEE conferences. He is active reviewer for IEEE Trans. and Letters, editor for the Journal of Information Technology and software feature topic issue Engineering (JITSE) and the International Journal of Applied Control, Electrical and Electronics Engineering (IJACEEE). He is a guest editor for IEEE Commun. feature topic issue on emerging applications, services and engineering for cognitive cellular systems to appear in 2015. He has been awarded the 2014 IEEE ComSoc Fred W. Ellersick Prize Award. His research team is currently involved in EU funded iJoin project which specifically focuses on backhaul solutions.
Mounir Ghogho received the M.S. de-

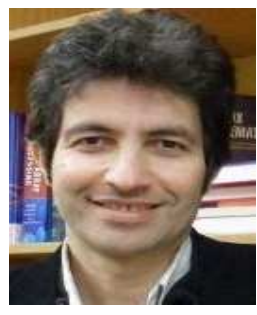
gree in 1993 and the $\mathrm{PhD}$ degree in 1997 from the National Polytechnic Institute of Toulouse, France. He was an EPSRC Research Fellow with the University of Strathclyde, Glasgow (Scotland), from September 1997 to November 2001. Since December 2001, he has been a faculty member with the school of Electronic and Electrical Engineering at the University of Leeds (England), where he is currently a Professor. He is also currently a Professor at the International University of Rabat (Morocco). He served as an Associate Editor of the IEEE Signal Processing Letters from 2001 to 2004 and the IEEE Transactions on Signal Processing from 2005 to 2008 . He is currently an associate editor of the Elsevier Digital Signal Processing journal. He served as a member of the IEEE Signal Processing Society SPCOM Technical Committee from 2005 to 2010, a member of IEEE Signal Processing Society SPTM Technical Committee from 2006 to 2011, and is currently a member of the IEEE Signal Processing Society SAM Technical Committee. He was the general co-chair of the eleventh IEEE workshop on Signal Processing for Advanced Wireless Communications (SPAWC'2010), the technical co-chair of the MIMO symposium of IWCMC 2007 and IWCMC 2008, and a technical area co-chair of Eusipco 2008, Eusipco 2009 and ISCCSP'05. He is the general co-chair of Eusipco2013. He was the guest co-editor of the EURASIP Journal on Wireless Communications and Networking special issue on "Synchronization for Wireless Communications" and the Elsevier Physical Communications Journal special issue on "Advances in MIMO-OFDM". His research interests are in signal processing and communication. He held many invited scientist/professor positions at the US Army Research Lab (USA), Telecom Paris-Tech (France), University Carlos III of Madrid (Spain), ENSICA (France), Darmstadt Technical University (Germany), Beijing University of Posts and Telecommunication (China), and University Mohamed V (Morocco). He is the Eurasip Liaison in Morocco. He was awarded the five-year Royal Academy of Engineering Research Fellowship in September 2000. 


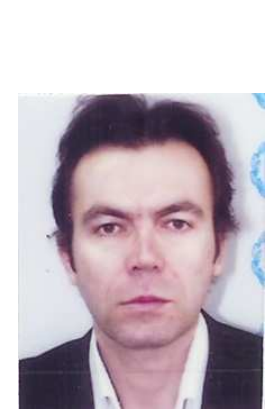

Athanasios V. Vasilakos is recently Distinguished Professor with the Kuwait University . He served or is serving as an Editor for many technical journals,such as the IEEE TRANSACTIONS ON NETWORK AND SERVICE MANAGEMENT; IEEE TRANSACTIONS ON CLOUD COMPUTING, IEEE TRANSACTIONS ON INFORMATION FORENSICS AND SECURITY,IEEE TRANSACTIONS ON CYBERNETICS; IEEE TRANSACTIONS ON NANOBIOSCIENCE; IEEE TRANSACTIONS ON INFORMATION TECHNOLOGY IN BIOMEDICINE; ACM Transactions on Autonomous and Adaptive Systems; the IEEE JOURNAL ON SELECTED AREAS IN COMMUNICATIONS . He is also General Chair of the European Alliances for Innovation(www.eai.eu ).

Desmond C. McLernon received the B.Sc. in electronic and electrical engi-

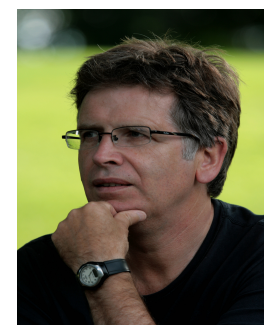
neering and the M.Sc. in electronics from Queen's University of Belfast, N. Ireland. He then worked towards the research and development of radar systems with Ferranti Ltd. in Edinburgh, Scotland and later joined Imperial College, University of London to obtain the Ph.D. degree in signal processing. After first lecturing at South Bank University, London, UK, he moved to the School of Electronic and Electrical Engineering at the University of Leeds, UK, where he is currently a Reader in Signal Processing and a Post Graduate Research Tutor. His research interests are broadly within the domain of signal processing for wireless communications systems (in which discipline he has published over 265 international journal and conference papers). He has supervised over $30 \mathrm{PhD}$ students, given invited talks in the UK and abroad, is the associate editor of the UK IET journal "Signal Processing" and engineering editor of the "Arabian Journal for Science and Engineering (Springer Publishing)". He has been a member of various international conference organization committees - more recently IEEE workshop on Signal Processing Advances in Wireless Communications (SPAWC 2010, Marrakech), European Signal Processing Conference (EUSIPCO) 2013, IET Conference on Intelligent Signal Processing (London, 2013 and 2015) and IEEE Globecom 2014, 2nd Workshop on Trusted Communications with Physical Layer Security (Austin, Texas).
Khalid A. Qaraq was born in Bethlehem. Dr Qaraqe received the B.S. degree in EE from the University of Technology, in 1986, with honors. He received the M.S. degree in EE from the University of Jordan, Jordan, in 1989, and he earned his Ph.D. degree in EE from Texas A\&M University, College Station, TX, in 1997. From 1989 to 2004 Dr Qaraqe has held a variety positions in many companies and he has over 12 years of experience in the telecommunication industry. Dr Qaraqe has worked for Qualcomm, Enad Design Systems, Cadence Design Systems/Tality Corporation, STC, SBC and Ericsson. He has worked on numerous GSM, CDMA, WCDMA projects and has experience in product development, design, deployments, testing and integration. Dr Qaraqe joined the department of Electrical and Computer Engineering of Texas A\&M University at Qatar, in July 2004, where he is now a professor. Dr Qaraqe research interests include communication theory and its application to design and performance, analysis of cellular systems and indoor communication systems. Particular interests are in mobile networks, broadband wireless access, cooperative networks, cognitive radio, diversity techniques and beyond $4 \mathrm{G}$ systems. 